\title{
Efficient Methods for Checking Integrity: An Integrated Spreadsheet Engineering Methodology (ISEM)
}

\author{
Kamalasen Rajalingham, David Chadwick, Brian Knight, \\ Dilwyn Edwards \\ Information Integrity Research Centre \\ School of Computing \& Mathematical Sciences \\ University of Greenwich \\ Wellington Street, Woolwich, London SE18 6PF, United Kingdom \\ k.rajalingham@gre.ac.uk
}

Key words: $\quad$ Spreadsheets, Errors, Systems Development Life Cycle, Software Engineering, Auditing

\begin{abstract}
:
This paper describes an approach to the provision of an Integrated Spreadsheet Engineering Methodology (ISEM). The proposed methodology is mainly based on the classical systems development life cycle. It addresses the widespread problem of spreadsheet errors and is an extension to published work by Chadwick et al (1997), Rajalingham \& Chadwick (1998) and Rajalingham et al (1999). This methodology also helps in training users in the process of spreadsheet building.

Although there are variations of the life cycle for systems development, they are fundamentally similar to each other. The Integrated Spreadsheet Engineering Methodology (ISEM) is primarily based on the systems development life cycle described by Aktas A Z (1987) and approaches recommended by other authors. Numerous approaches are incorporated into this framework, making it a highly integrated methodology for spreadsheet design and development.

Apart from the concepts and principles borrowed from the above methods, the Integrated Spreadsheet Engineering Methodology (ISEM) also contains new developments in the research into integrity control in spreadsheets. The potential for the proposed methodology to address the different types of spreadsheet errors is also discussed.
\end{abstract} corrected. The Erratum to this chapter is available at DOI: 10.1007/978-0-387-35501-6_14 


\section{INTRODUCTION}

The problem of data integrity in spreadsheet models has attracted much attention and concern over the years. Although much attention has been devoted to the verification and validation of information processes in general, there has been relatively little research undertaken into developing satisfactory solutions to the problem of spreadsheet errors. As a result, this phenomenon continues to exist, having a serious effect on businesses and costing them a lot of money.

Numerous publications over the years have confirmed and provided sufficient evidence that spreadsheet errors have adversely affected businesses. The phenomenon of spreadsheet errors can be explored or investigated from three different perspectives. They are as follows:

- the frequency of the occurrence of the errors;

- the real-life consequences of spreadsheet errors;

- the actual errors themselves.

\subsection{Frequency of Spreadsheet Errors}

This aspect of the spreadsheet integrity problem has been given adequate attention. As a result, most of the literature on this issue concern the frequency of the occurrence of spreadsheet errors. Numerous related experiments and studies have been carried out in the past and sufficient information is presently available.

Based on material from a wide variety of publications, Panko and Halverson (1996) have organised the research findings into an excellent compilation statistics on the frequency of spreadsheet errors. Large and well-known auditing firms such as KPMG Management Consulting and Coopers \& Lybrand have also reported that spreadsheet errors are occurring at appalling rates. These organisations constantly audit very large numbers of spreadsheet models from various clients. KPMG Management Consulting (KPMG, 1997) and Coopers \& Lybrand (Ward, 1997) have reported that more than $90 \%$ of the spreadsheet models they inspected contained errors.

The conclusion that can be reached after examining all these reports is that the rate of occurrence of spreadsheet errors is in fact significantly high.

\subsection{The Real-life Consequences}

The second aspect of the spreadsheet integrity phenomenon concern the real-life impact and consequences of spreadsheet errors. The purpose of investigating the negative effect of these errors on businesses is to enable better understanding of the magnitude and seriousness of the problem of spreadsheet errors. 
Numerous recent publications have indicated the seriousness of spreadsheet errors and their adverse impact or potential impact on businesses, quoting many relevant cases. It must however be noted that these are just reported cases. There must be many other similar cases that have not been brought to public attention due to fear that they might affect the reputation of the company involved.

Some of the reported cases are given in Chadwick et al (1997), Rajalingham \& Chadwick (1998) and Rajalingham et al (1999). Although many of these cases are not based on formal research, they do show that spreadsheet errors are deemed serious enough to be reported in the general business and computing press. They also show the extent of the damage that can be caused. If this situation prevails, companies will be losing a lot of money due to poor decisions made based on the unreliable spreadsheet figures.

\subsection{The Specific Errors and Flaws Committed}

This is an area that has not at all been adequately explored or discussed in publications on the problem of spreadsheet errors. Four research papers that have addressed this issue and analysed specific types of spreadsheet errors from business and academia are Panko and Halverson (1996), Chadwick et al (1997), Rajalingham \& Chadwick (1998), and Rajalingham et al (1999).

The outcome of research into specific types of spreadsheet errors is the provision of a more comprehensive classification or taxonomy of spreadsheet errors. The elements of this classification of spreadsheet errors are presented and elaborately discussed by Rajalingham \& Chadwick (1998) and Rajalingham et al (1999), supported by relevant examples.

Figure 1 shows an improved version of the model, by Rajalingham \& Chadwick (1998) and Rajalingham et al (1999). Referring to figure 1, the elements of the taxonomy (UPPER CASE) are given, accompanied by the specific types of spreadsheet errors (lower case italics) they are associated with.

A careful analysis of these different aspects of the spreadsheet integrity problem clearly shows the seriousness of the situation. As such, new methods and techniques are required to control the integrity of spreadsheet errors and to reduce the occurrence of these errors. 


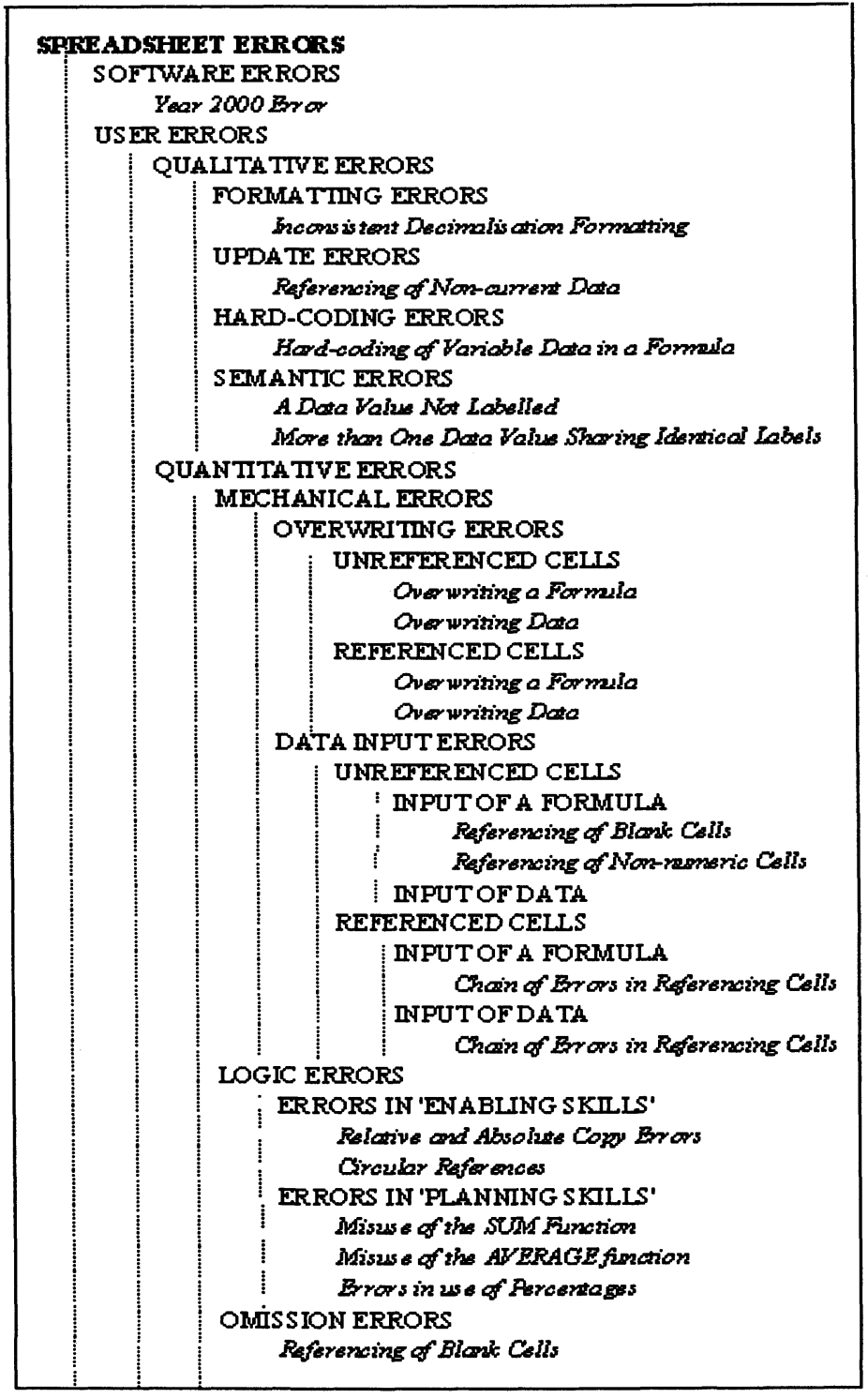

Figure 1. Specific Types of Spreadsheet Errors 


\section{THE INTEGRATED SPREADSHEET ENGINEERING METHODOLOGY (ISEM)}

This section presents a comprehensive methodology for spreadsheet development based on various approaches, methods, tools and techniques proposed in publications as well as original work done by the authors of this paper. This is in line with the constant call by numerous authors to impose some discipline and structure in spreadsheet development, as a step towards controlling the integrity of spreadsheet models. The methodology consists of five main stages:

Stage 1: Planning

Stage 2: Analysis

Stage 3: Design

Stage 4: Implementation

Stage 5: Maintenance

\subsection{Rationale for a Structured Approach or Methodology}

An investigation carried out into formal development methods used in industry revealed that large scale software developments by professional computing staff were subject to formal development methods and monitored by auditors for errors throughout their life-history. On the other hand, small scale applications, such as spreadsheet models, were not subjected to such methodologies or structured methods (Chadwick et al, 1997).

It has been observed for several years at the University of Greenwich, UK that students keep making similar mistakes and errors when developing spreadsheet models. However, presently available literature on spreadsheet errors do not offer sufficient or effective methods, tools, techniques or guidelines that can be used in the process of constructing spreadsheets. It was therefore realised that better methods were needed in order to aid students in making fewer errors whilst building a spreadsheet and to aid the lecturer in assessing the students' spreadsheet for correctness.

Research findings over the last few years clearly show the need for a structured approach or discipline for spreadsheet development. This is mainly due to the absence of recognised methods for spreadsheet design and development. Many authors on the subject have recently called for a disciplined or structured approach to spreadsheet building. Panko and Halverson (1996, January) distinctly state that there is a need to adopt strict programming disciplines in dealing with complex spreadsheets. The journal paper by Panko (1998) also says that there is an obvious need to begin adopting traditional programming disciplines due to the similarity between spreadsheet errors and programming errors. The paper also states that there is far too little knowledge of spreadsheet errors, which implies that much more research has to be undertaken into spreadsheet errors. 
Based on various published reports, we can come to the conclusion that there is a need for the adoption of a structured methodology in spreadsheet development. This will certainly help address the currently major problem of spreadsheet errors.

\subsection{Elements of the Methodology}

The unique feature of this methodology is that it brings together various approaches, most of which have already been discussed in previous publications. The basic framework or foundation for this methodology is the classical systems development life cycle given by Aktas (1987). The steps and stages within this life cycle are now being proposed for spreadsheet systems as well. It should also be noted that the proposed methodology caters for large and complex spreadsheet systems. As such, some of the steps can be omitted when building small or simple systems.

\section{STAGE 1: PLANNING}

Step 1: Request for construction of the spreadsheet system

The process of building a spreadsheet system or modifying an existing one is initiated through a request by management.

Step 2: Initial investigation of the spreadsheet system

The main requirements for the spreadsheet system are established. There is also a study of the spreadsheet system's interfaces with other elements such as hardware, people, databases and other software. This encompasses requirements gathering at the system level with a small amount of top-level design and analysis.

Step 3: Feasibility study of the spreadsheet system

This step is important as it establishes whether the proposed system is in fact best developed using spreadsheet software. If certain requirements cannot be met by the spreadsheet software, other alternatives should be considered, such as a database system. The analyst should also determine if expertise to build the spreadsheet system is available.

\section{STAGE 2: ANALYSIS}

The requirements gathering process is intensified and focused specifically on the spreadsheet model. 
Step 1: Redefine/define the spreadsheet problem

The problem to be put on the computer and the nature of the spreadsheet model to be built has to be clearly understood. This includes the information domain for the system as well as the required functions, performance, and interfacing.

The main requirements of a spreadsheet system normally take the form of operations on data values. These operations are implemented as formulae. As such formulae lie at the heart of spreadsheet modelling and they are the most important elements of a spreadsheet model. This is also because formulae are the ones that actually calculate and provide useful results based on raw, user-entered data values. Research findings from various sources have indicated that a majority of errors committed in spreadsheets are formula-based errors. They are committed either due to a lack of understanding of the underlying algorithm or carelessness in entering the right cell addresses.

Rajalingham et al (1999) have proposed a tree-structure for visually representing elements of a spreadsheet formula. In the same way that a computer program can be broken down into smaller parts and represented in the form of a tree, elements of a spreadsheet formula can also be represented in a similar manner. This helps confirm our understanding of the structure and components of a particular formula so that the requirements of the system are effectively implemented. This technique has also been described in detail by Chadwick et al (1999), who have used the method to visually represent spreadsheet models in tree-like structures.

The tree represents all the elements of a particular formula (data values, hard-coded constants, cells referenced, binary operators and built-in functions). Based on the spreadsheet model shown in figure 2, a selected formula is represented in the form of a tree. This is shown in figure 3.

\begin{tabular}{|c|c|c|c|c|c|}
\hline C & D & E & $\mathrm{F}$ & $G$ & $\mathrm{H}$ \\
\hline & $\begin{array}{l}\text { Number of } \\
\text { Staff }\end{array}$ & DarWages \& & $\begin{array}{r}\text { Night } \\
\text { Wages f }\end{array}$ & Total Wages \& & Average Wage \\
\hline Grade 1 & 1 & 17700.50 & 0.00 & 17700.50 & 17700.50 \\
\hline Grade 2 & 3 & 45540.00 & 1400.55 & 46940.55 & 15646.85 \\
\hline Grade 3 & 9 & 122340.00 & 2000.00 & 124340.00 & 13815.56 \\
\hline Grade 4 & 12 & 102350.25 & 0.00 & 102350.25 & 8529.19 \\
\hline Grand Total & 25 & 2879030.75 & 3400.55 & 291331,30 & 11653,25 \\
\hline
\end{tabular}

Figure 2. Staff Budget Model 


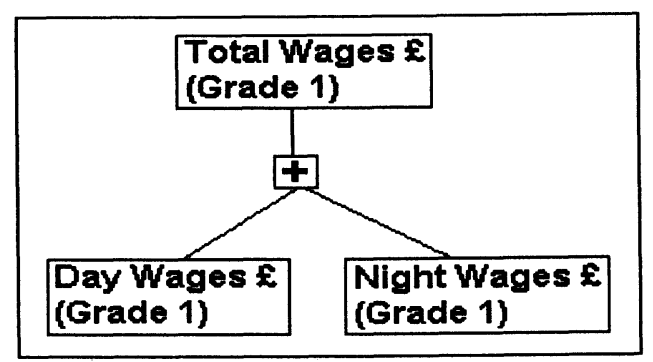

Figure 3. A Selected Formula Represented in Tree Form

Step 2: Understand existing Spreadsheet System (if there is one)

Often times, especially in large organisations, there is a need to modify or improve an existing spreadsheet system rather than developing one from scratch.

Step 3: User requirements and constraints of the spreadsheet system

A good understanding of the requirements of users and constraints to be imposed within the system is important and should be carefully considered.

\section{STAGE 3: DESIGN}

The design process translates requirements into a representation of the system that can be assessed for quality before system building begins. This stage consists of two main phases, namely, logical design and physical design.

Step 1: Logical design

This is a high-level representation of the system, its requirements, data structure and operations. The location of the various elements of the model on the spreadsheet is not specified at this stage. The operations to be performed (functions) are represented as general mathematical formulae using relevant labels.

Based on methods proposed by Rajalingham \& Chadwick (1998) and Rajalingham et al (1999), it is important to uniquely define elements of a spreadsheet model. This means, that every data value or formula must be associated with a unique label or a unique pair of column and row labels. The label(s) must be semantically consistent with the meaning of the data value/formula in real life. Figure 4 (Wood, 1996) shows a spreadsheet model having an ambiguity problem due to the absence of this useful constraint. This problem and the example have been discussed by Rajalingham et al (1999). 


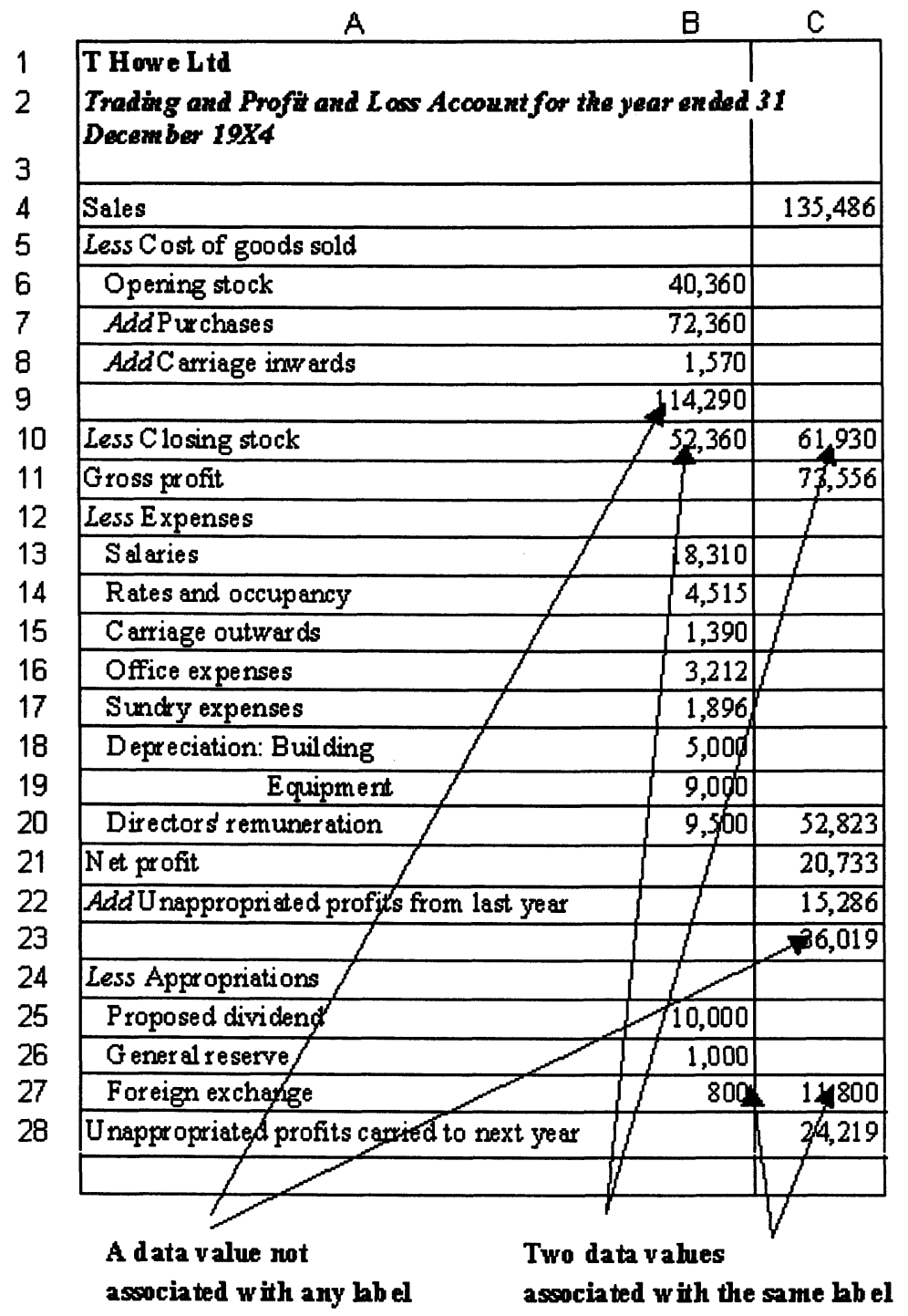

Figure 4. Spreadsheet Model with an Ambiguity Problem

Based on the model shown in figure 4, it can be observed that certain data values are not associated with any labels e.g. cells B9 $(114,290)$ and C23 $(36,019)$. Apart from that, certain labels are placed on the same row as two different values, not distinguished by a column label e.g. cells B27 and C27, both being on the same row and labelled foreign exchange. Only after carefully studying the model it is known 
that cell C27 actually represents the 'total of appropriations'. Although the meaning of these values can be understood by carefully studying the model, it is not immediately obvious, especially to someone who is not familiar with such models.

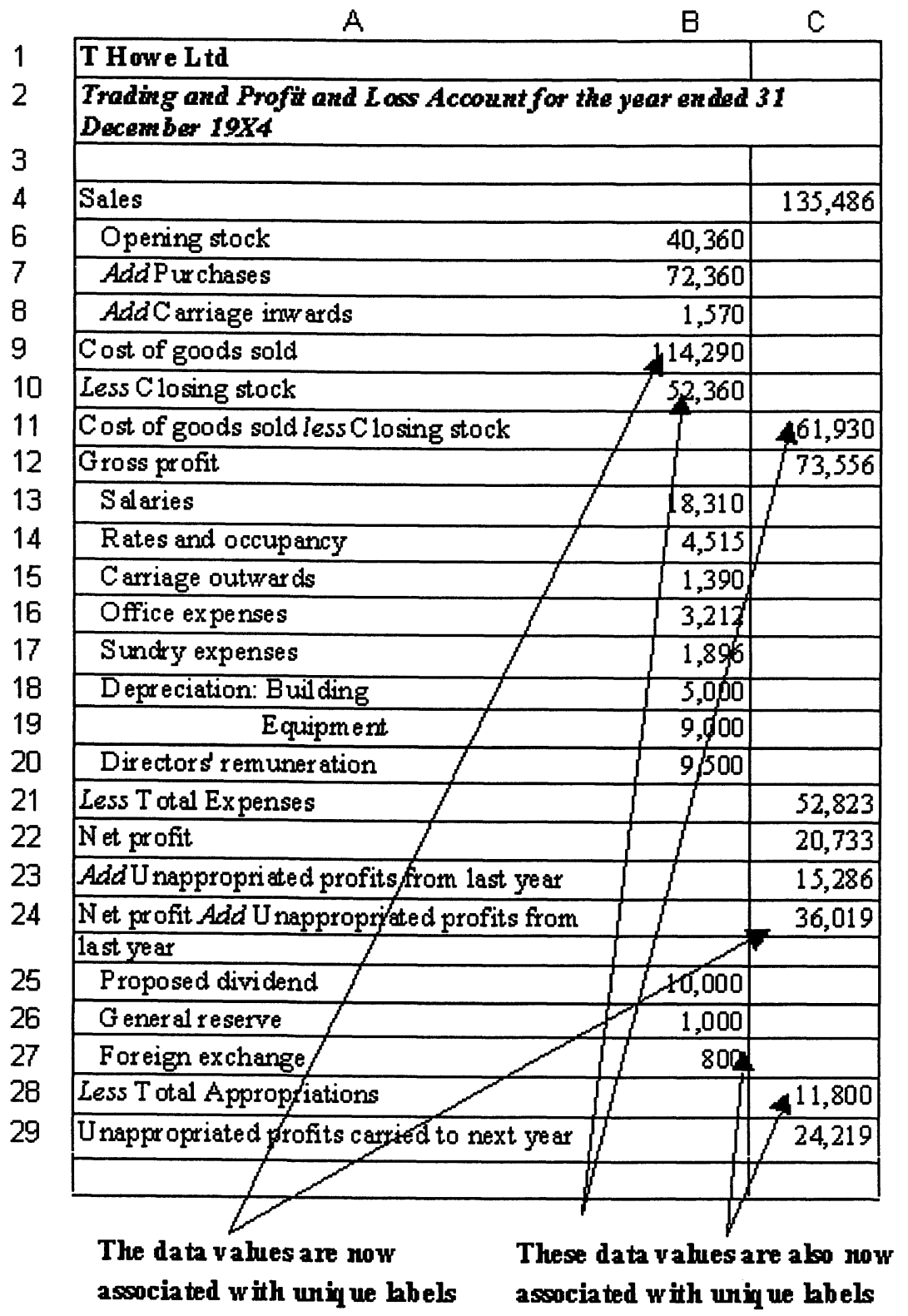

Figure 5. The Improved Spreadsheet Model 
Figure 5 shows the improved model with the ambiguity problem removed. This has been accomplished by imposing the uniqueness constraint upon the elements of the spreadsheet model.

A frequent error committed by users is the accidental overwriting of formulae. This is usually due to the fact that data values and their referencing formulae are placed close to each other and users are sometimes unable to distinguish between the two. The technique proposed to overcome this problem is to put all user-entered data values into a separate matrix on the spreadsheet. The original, complete model only holds absolute copies of the corresponding values in the data matrix. It is also protected as a pre-caution against any overwriting of data. This technique is employed in the methodology for spreadsheet development (based on data structure) presented by Chadwick et al (1999).

This strategy is similar to the method introduced by DiAntonio (1986). DiAntonio's method advocates the isolation of facts by splitting the spreadsheet into two parts, one for the facts and one for the solution. The solution is formatted or designed and it uses data from the facts part of the spreadsheet.

The proposed technique of separating data values is illustrated based on the spreadsheet model shown in figure 2. The data values are identified and organised into a separate data matrix. This is shown in figure 6.

\begin{tabular}{|c|c|c|c|c|c|c|}
\hline & $1 \mathrm{mod}$ & cted) & & & & \\
\hline C & D & $\mathrm{E}$ & $\mathrm{F}$ & $G$ & $\mathrm{H}$ & \\
\hline & $\begin{array}{r}\text { Number of } \\
\text { Staff }\end{array}$ & DerWages $\varepsilon$ & $\begin{array}{r}\text { Night } \\
\text { Wages } 5\end{array}$ & Total Wages f & Average Wage & 5 \\
\hline Grade 1 & 1 & 17700.50 & 0.00 & 17700.50 & 17700.50 & 6 \\
\hline Grade 2 & 3 & 45540.00 & 1400,55 & 46940.55 & 15646.25 & 7 \\
\hline Grade 3 & 9 & 122340.00 & 2000.00 & 124340.00 & 13815.56 & 8 \\
\hline Grade 4 & 12 & 102350.25 & 0.00 & 102350.25 & 8529.19 & 9 \\
\hline Grend Total & 25 & 287930.75 & 3400.55 & 291331.30 & 11653.25 & 10 \\
\hline
\end{tabular}

\begin{tabular}{|c|c|c|c|c|}
\hline \multicolumn{5}{|c|}{ Data matrix } \\
\hline$c$ & D & E & $\mathrm{F}$ & \\
\hline & $\begin{array}{r}\text { Number of } \\
\text { Staff }\end{array}$ & DarWages $\tilde{E}$ & $\begin{array}{r}\text { Night } \\
\text { Wages }\end{array}$ & 13 \\
\hline Grade1 & 1 & 17700.50 & 0.00 & 14 \\
\hline Grade 2 & 3 & $45540 . \infty$ & 1400.55 & 15 \\
\hline Grade 3 & 9 & 122340.00 & 2000.00 & 16 \\
\hline Grade 4 & 12 & 102350.25 & 0.00 & 17 \\
\hline
\end{tabular}

Figure 6. Formation of a data matrix

Step 2: Physical design

Based on the logical design of the spreadsheet model, the location of the various elements or components of the model on the physical spreadsheet is determined. 
This is a low-level design of the spreadsheet model. The functions that operate on data values are specified using the right syntax and in terms of cell addresses as well, rather than just labels.

Figure 3 shows a conceptual or logical representation of a spreadsheet formula in tree form. Tree structures can also be constructed to represent the physical aspect of a formula, using cell addresses. As all functions are of the same form, = name (argument1, argument2 ... ), we can even represent each function in the form of a tree (not necessarily a binary tree). The root would now contain the function name while each argument would form a node. Examples (Rajalingham at el, 1999) are given in figures $7 a$ and $7 b$.
E.g.
$=\mathrm{A} 1+\mathrm{B} 1$
2. $=\mathrm{A} 1 / \mathrm{B} 1$ i.e. $\mathrm{A} 1 \div \mathrm{B} 1$
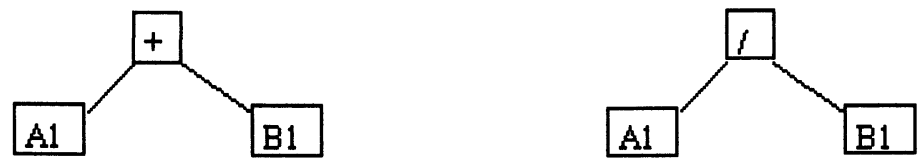

3. $=A 1 \sim(B 1 / B 2)$ i.e. $\mathrm{B}_{2} \sqrt{A 1}^{\mathrm{B} 1}$

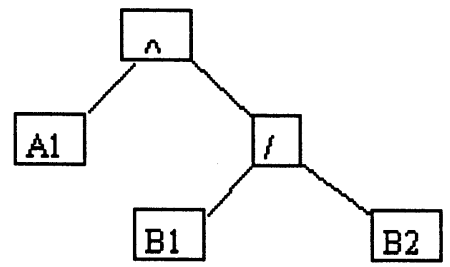

Figure 7a. Examples of Formulae in Tree Form
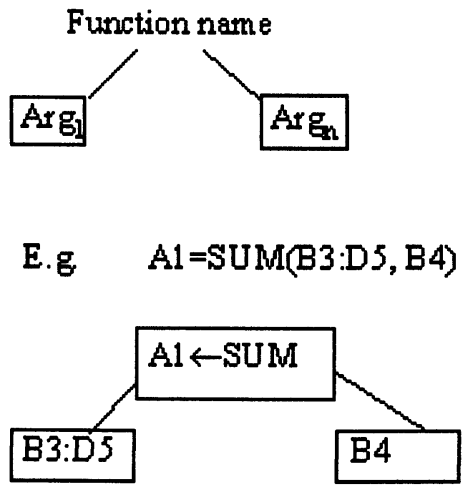

Figure $7 b$. Example of a Function in Tree Form 


\section{STAGE 4: IMPLEMENTATION}

Step 1: Spreadsheet system building

The physical design of the spreadsheet model is actually implemented using a particular spreadsheet package e.g. Microsoft Excel, Lotus 123 etc.

Step 2: Testing of the spreadsheet system

The testing process focuses on whether or not all the requirements of the system are met. The data values are checked for omitted or redundant items. All data values referenced by some formula must be present in the model. The formulae are checked to make sure that they produce the desired results. The tests uncovers errors and ensure that defined input will produce actual results that agree with required results.

Three important tools that can be used to aid model testing are the Microsoft Excel Audit Tool (Chadwick et al, 1997), Spreadsheet Professional for MS Excel by Spreadsheet Innovations (Rajalingham \& Chadwick, 1998) and the Checklist Approach (Kruck, 1998).

The audit tool which is part of the Microsoft Excel software enables the user to easily trace the precedents or dependants of any cell. The precedents of a cell are the cells referenced by it while the dependants of a cell are the cells that reference it. When tracing the precedents of a cell, an arrowed line is drawn from each precedent cell, poiting to the dependant cell. On the other hand, when tracing the dependants of a cell, an arrowed line is drawn from that cell pointing to each of its dependant cells.

E.g. Referring to figure 8, the precedents of cell E13 are cells E8 and E9, while the dependant of cell E8 is cell E13.

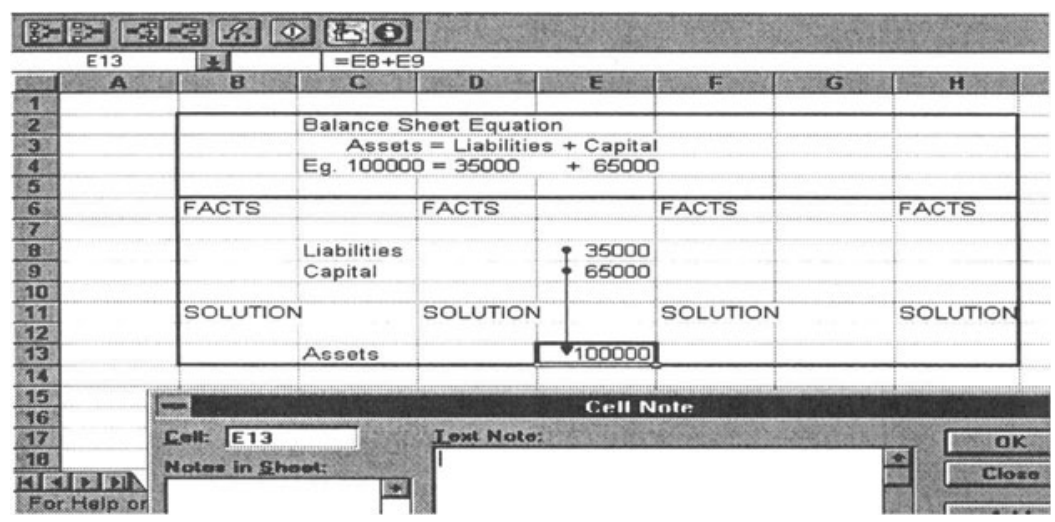

Figure 8. MS Excel Audit Tool 
Apart from that, the audit tool also offers a facility for the user to attach a note or description to a cell.

The second tool is Spreadsheet Professional for Microsoft Excel by Spreadsheet Innovations. This tool, which is an add-on to Microsoft Excel, has served as a rather useful auditing tool. It has various functions to help detect errors in the spreadsheet model. Among the significant functions of this tool are the calculation checker and the cell translation facility.

The Calculation Checker enables the user to view the contents, potential error and precedents of a formula cell. Based on the spreadsheet model shown in figure 2, if a 'calculation check' is done on cell $\mathrm{H} 10$ and the 'Show Precedents' option is subsequently selected, the precedents of cell $\mathrm{H} 10$ are displayed as shown in figure 9.

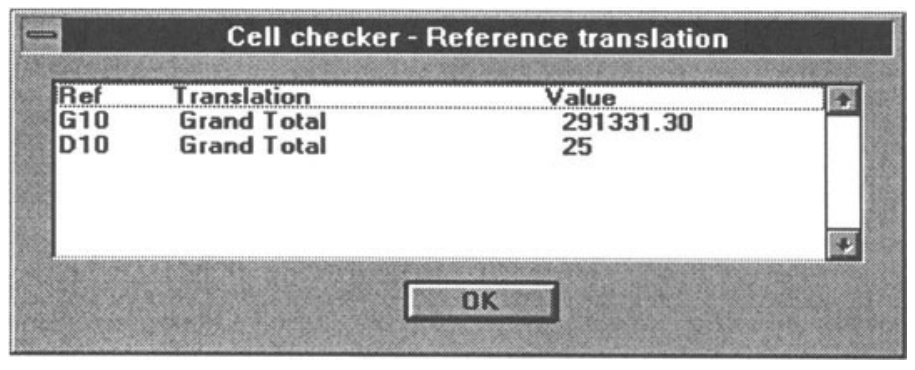

Figure 9. Calculation Checker

The translation for each precedent (cell or range) is given in terms of location, corresponding row heading and values.

The result of a 'Cell Translation' for cell E10 would produce information as given in figure 10.

$$
\begin{array}{ll}
\text { Sheetl!E10 } & =\text { SUM (E6:E9 } \\
\text { Grade T otal } & =\text { SUM (Grade 1: Grade 4) } \\
287930.75 & =\text { SUM (17700.50:102350.25) }
\end{array}
$$

Figure 10. Cell Translation

The formula in the cell is first translated into a form where each cell address is replaced with its corresponding row heading and then presented in a form where each cell address is replaced with its corresponding value. 
Chadwick et al (1997, December) has proposed the 3A's (appropriateness, accuracy, about-right) Approach for spreadsheet auditing. This method can be used to test the spreadsheet model. The detailed version of the methodology can be obtained from the source paper. The following are the elements of Chadwick et al's 3A's approach:

Step 1: Checking the appropriateness of the formula applied, from a logical point of view, based on the underlying business model.

Step 2: Checking the accuracy of the formula entered based on a correct interpretation of the data model.

Step 3: Checking if the resulting numeric value of the cell is about right.

Step 3: Document the spreadsheet system/program

Documentation may be included in a special area of the spreadsheet (on-line) or may be prepared in hard-copy form. It includes the program rationale and objectives. The author name and date prepared should also be part of the documentation. In addition to that, there should also be a log of all changes made to the spreadsheet model.

The tree method described under stage 2 (step 1) and stage 3 (step 2) can also be used as a means of documenting the spreadsheet model. The tree-structure facilitates better comprehension of the spreadsheet model. The model can be represented as an interconnection of formula trees.

Step 4: Operation of spreadsheet system

Step 5: Post-implementation review of the spreadsheet system

\section{STAGE 5: MAINTENANCE}

The delivered spreadsheet system will undoubtedly have to undergo changes, due to advances in spreadsheet software as well as changing user requirements. Changes will also have to be made because of quantitative and qualitative errors that have been encountered. The system may also need to be adapted to accommodate changes in the external environment. System maintenance reapplies each of the preceding life-cycle stages and steps.

\section{CONCLUSION}

The Integrated Spreadsheet Engineering Methodology (ISEM) is mainly based on the classical systems development life cycle presented by Aktas (1987). This life cycle is based on a structured approach. Adopting such an approach to spreadsheet design and development imposes some discipline in the spreadsheet building process. This is exactly what has been deemed important and necessary by many authors on the subject of spreadsheet errors and integrity control of spreadsheet models. 


\section{ST AGE 1: PLANNING}

Step 1: Request for construction of the spreadsheet system

Step 2: Iritial investigation of the spreadshe et system

Step 3: Feasibility study of the spreadsheet system

ST AGE 2: ANAL YSIS

Step 1: Redefine/de fine the spreadsheet problem

Step 2: Understand existing Spreadsheet System (if there is one)

Step 3: U ser requirem ents and constraints of the spreadsheet system

ST AGE 3: DESIGN

Step 1: Logical de sign UNIQUE DEFINITION OF

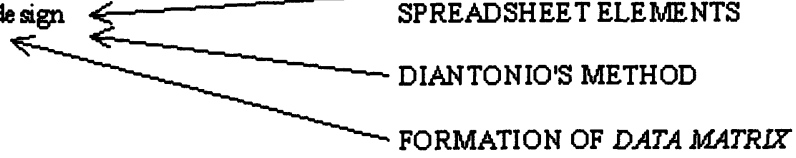

Step 2: Physical design TREE METHOD

ST AGE 4: IMPLEMENTATION

Step 1: Spreadsheet system building

Step 2: Testing of the spreadsheet system THE CHECKLIST APPROACH

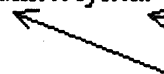
MS EXCEL AUDIT TOOL SPREADSHEET PROFESSIONAL FOR MS EXCEL THE 3A'S APPROACH

Step 3: Document the spreadsheet TREE METHOD system/program

Step 4: Operation of spreadsheet system

Step 5: Post-implementation review of the spreadsheet system

\section{ST AGE 5: MAINTENANCE}

Figure 11. The Integrated Spreadsheet Engineering Methodology (ISEM) 
The proposed methodology has incorporated various tools, techniques, methods and principles for spreadsheet development, already published as well as new developments in the research into improved methods for integrity control in spreadsheet models. The integrated methodology also has the potential to prevent and reduce most of the errors given in the taxonomy of errors in section 1.3. This has been discussed within the description of the stages and steps of the methodology, in the previous section.

An approach based on this methodology is presently being tried out with students at the University of Greenwich (UK) with some success.

\section{REFERENCES}

Aktas A Z (1987). Structured Analysis \& Design of Information Systems. PrenticeHall.

Chadwick D, Knight J \& Clipsham P (1997). Information Integrity In End-user Systems. Proceedings of the IFIP TC-11 Working Group 11.5 First Working Conference on Integrity and Internal Control in Information Systems, Zurich, Switzerland (December, 1997).

Chadwick D, Rajalingham K, Knight B \& Edwards D (1999). A Methodology for Spreadsheet Development Based on Data Structure. Paper No: 99/IM/50 (June, 1999). CMS Press.

DiAntonio A E (1986). Spreadsheet Applications. Prentice-Hall.

KPMG Financial Modelling Department (1997). Executive Summary: Financial Model Review Survey. KPMG Management Consulting (London).

Kruck S (1998). Towards a Theory of Spreadsheet Accuracy: An Empirical Study. Paper delivered at the Decision Sciences Institute, Las Vegas, USA (November, 1998).

Panko R R \& Halverson R P Jr. (1996). Spreadsheets on Trial: A Framework for Research on Spreadsheet Risks. Proceedings of the Twenty-Ninth Hawaii International Conference on System Sciences, Maui, Hawaii (January, 1996).

Panko R R (1998). What We Know About Spreadsheet Errors. Journal of End User Computing's Special issue on Scaling Up End User Development, Spring 1998.

Rajalingham K \& Chadwick D (1998). Integrity Control of Spreadsheets: Organisation \& Tools. Proceedings of the Second Annual IFIP TC-11 Working 
Group 11.5 Working Conference on Integrity and Internal Control in Information Systems, Virginia, USA (19-20 November 1998). Chapman \& Hall.

Rajalingham K, Chadwick D, Knight B \& Edwards D (1999). An Approach to Improving the Quality of Spreadsheet Models. Proceedings of the Seventh International Conference on Software Quality Management SQM'99, Southampton, UK, March 1999. British Computer Society.

Ward M (1997). Fatal Addition. New Scientist (16 August 1997).

Wood F (1996). Business Accounting 1 (7th Edition). Pitman Publishing. 